\title{
Prognostic value of the immunohistochemical expression of vascular endothelial growth factors in malignant salivary gland neoplasms: a systematic review and meta-analysis
}

\author{
Erison Santana dos Santos ${ }^{1}$, Joab Cabral Ramos ${ }^{1}$, Ana Gabriela Costa Normando ${ }^{1}$, Adriana Franco Paes Leme ${ }^{2}$ \\ ${ }^{1}$ DDS, MSc, PhD Student. Department of Oral Diagnosis, Piracicaba Dental School, University of Campinas, Piracicaba, Brazil \\ ${ }^{2}$ DDS, MSc, PhD, Professor. Brazilian Bioscience National Laboratory, Brazil Center of Research in Energy and Materials, \\ Campinas, Brazil
}

Correspondence:

Departamento de Diagnóstico Oral, Área de Patologia Oral

Faculdade de Odontologia de Piracicaba, Universidade Estadual de Campinas (UNICAMP)

Av. Limeira, 901, CEP: 13.414-903, Piracicaba, São Paulo (SP), Brasil

erisonsantosupe@hotmail.com

Received: 07/05/2020

Accepted: 07/01/202

dos Santos ES, Ramos JC, Normando AGC, Leme AFP. Prognostic value of the immunohistochemical expression of vascular endothelial growth factors in malignant salivary gland neoplasms: a systematic review and meta-analysis. Med Oral Patol Oral Cir Bucal. 2021 Mar 1;26 (2):e126-35.

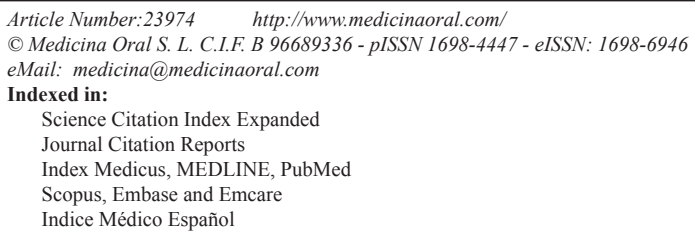

\begin{abstract}
Background: The immunohistochemical expression of vascular endothelial growth factor is a prognostic marker in several cancer types. In salivary gland tumors, the association between vascular endothelial growth factor and prognosis remains unclear. The purpose of this study was to perform a systematic review and meta-analysis to assess whether the immunohistochemical expression of vascular endothelial growth factor in patients with salivary gland neoplasms presents prognostic value.

Material and Methods: Immunohistochemical studies assessing the predictive value of vascular endothelial growth factor in salivary gland neoplasms were systematically reviewed using PubMed, Scopus, Embase, Cochrane Library, and Web of Science databases. It was assessed any survival rates. The fixed-effect model with an adjusted hazard ratio (HR) and 95\% confidence intervals (95\% CI) as effect measures were performed in the meta-analysis. The Quality in Prognosis Studies (QUIPS) tool was used to assess the quality of the included studies, and the evidence quality was assessed by the Grading of Recommendation, Assessment, Development, and Evaluation (GRADE) system.

Results: The immunohistochemical overexpression of vascular endothelial growth factor in patients with salivary gland neoplasms was associated with shortened survival (HR=5.37, 95\% CI: $2.67-10.83, P=0.00001$ ). In addition, the presence of vascular endothelial growth factor was tightly associated with tumor size, lymph node metastasis, clinical stage, perineural invasion, vascular invasion, poor local control of the disease, and recurrence.

Conclusions: The immunohistochemical overexpression of vascular endothelial growth factor in patients with salivary gland neoplasms has prognostic value and was associated with decreased survival time. However, more primary well-designed studies are necessary to increase the level of evidence.
\end{abstract}

Key words: Salivary gland neoplasms, salivary glands, head and neck neoplasms, vascular endothelial growth factors, prognosis. 


\section{Introduction}

Angiogenesis is defined as the development of new blood vessels from the preexisting vascular beds and plays an essential role in the tumor's maintenance, growth, and progression (1). A range of molecules such as cytokines, proteins, and growth factors tightly regulate this process $(1,2)$. Among various angiogenic factors, the most studied is the vascular endothelial growth factor (VEGF) due to its capacity to initiate and regulate the process of angiogenesis. This biologic event is complex, and it has been shown that hypoxia plays an essential role in the initiation of angiogenesis (1-3). The lack of oxygen leads to the tumor's cells release pro-angiogenic factors such as VEGF (3). Besides, it has been demonstrated that hormones, growth factors, hypoglycemia, and altered expression of genes are capable of upregulating VEGF expression (3).

In the context of head and neck cancer, it has been demonstrated that VEGF overexpression is associated with poor overall survival (4). Nevertheless, it is still uncertain whether VEGF overexpression is a risk factor for shorter survival in patients with salivary gland neoplasms (SNGs). Salivary gland neoplasms are a diverse group of tumors with different clinical, biological, and molecular features that correspond to approximately $3 \%$ to $10 \%$ of the tumors of the head and neck region (5). Several studies have attempted to determine the prognostic importance of VEGF in these lesions; however, the results obtained are conflicting $(1,4,5)$. Therefore, the aim of this systematic review and meta-analysis was to assess the prognostic value of VEGF in patients with malignant SGNs.

\section{Material and Methods}

- Protocol and registration:

A search was carried out for any registered protocols on a similar topic in the International Prospective Register of Systematic Reviews (PROSPERO). No systematic review protocols were found in this database. Therefore, this review was registered in the PROSPERO platform under the identification number CRD42020181985. The systematic review was reported according to the Preferred Reporting Items for Systematic Reviews and Meta-Analyses (PRISMA) statement (6).

- Eligibility criteria

It was established a PECOS framework (Population, Exposure, Comparison, Outcomes, and Studies) according to PRISMA, that was used to formulate the focused question of the review, of which: P) patients with the diagnosis of malignant salivary gland neoplasms; E) analysis by immunohistochemical detection of VEGF C) patients with no history of malignant salivary gland neoplasms; O) survival analysis; S) observational studies (case-control, cross-sectional, or population-based) and/or randomized clinical trials. Articles were excluded by the following reasons: A) did not fit PECOS strategy; B) did not present prognostic value of VEGF on SGNs such as hazard ratio (HR) values and corresponding 95\% confidence intervals (CIs), disease-free survival (DFS), disease-specific survival (DSS), overall survival (OS) or any other survival analysis; C) in vitro or in vivo experimental studies, letters to the editor, short communications, personal opinions, conference abstracts, case reports, and reviews.

- Focused question

Is there any association between immunohistochemical detection of VEGF and overall survival in patients with malignant salivary gland neoplasms?

- Search strategy

A search was conducted on April 24, 2019, and adapted for each electronic database: PubMed, Scopus, Web of Science, Embase, and Cochrane Library. An additional gray literature search was performed on Google Scholar, Open Grey, and ProQuest Dissertation \& Theses Global. The search strategy was based on combinations of the following keywords: ("Vascular Endothelial Growth Factors"[MeSH] OR "VEGFs") AND ("Salivary Gland Neoplasms"[MeSH] OR "Salivary Gland Neoplasm" OR "Cancer of the Salivary Gland" OR "Salivary Gland Cancers" OR "Cancer of the Salivary Gland" OR "Salivary Gland Cancer" OR "Salivary Glands"[MeSH] OR "Salivary Gland"). All duplicate references were removed using a reference manager software (Rayyan QCRIR) (7). In addition, manual screening of the reference lists from the selected articles was performed to identify potentially relevant studies that could have been missed during the electronic database searches.

- Study selection and data extraction

The process of study selection was performed in two phases. First, titles and abstracts of all identified articles were screened by two independent reviewers (ESS and AGCN) using a standardized guide. This stage was conducted using Rayyan - a web and mobile app for systematic reviews available online (7). The same two authors read the full texts of the selected articles at phase one and excluded those that did not meet the inclusion criteria. Cohen's Kappa analysis was performed to a quantified agreement between the authors. Another author (AFPL) was involved in the case of doubts and conflicts. Two reviewers (ESS and AGCN) independently collected the data on study characteristics such as author, year of publication, country of the first author of the study, the number of samples enrolled, histologic type of SGN, follow up period, statistical method, and main results.

- Quality assessment

The recommendations of the Cochrane Prognosis Methods Group were followed and the Quality in 
Prognosis Studies (QUIPS) tool was used to assess the methodological quality of included studies $(8,9)$. The QUIPS tool assesses the risk of bias in prognostic studies by rating each article in six domains: study participation, study attrition, measurement of prognostic factors, measurement of outcomes, measurement of confounding, and statistical analysis and reporting. A judgment of the risk of bias on each domain of the tool was made from the extracted information, rated as "high," "moderate," or "low" risk. These judgments were realized by two authors (ESS and AGCN), independently and blindly. In case of disagreements, a third author (AFPL) was consulted.

- Summary of measures and synthesis of results

It was performed a meta-analysis of the survival rates following the appropriate Cochrane Guidelines for prognostic reviews (10). Review Manager 5.3 (RevMan 5.3, The Nordic Cochrane Centre, Copenhagen, Denmark) was used to construct the forest plots of the meta-analysis. The HR and 95\% CI were used to determine at a significance level of 5\%, according to the adjusted survival rates original values of the included articles. The heterogeneity between eligible studies was calculated by inconsistency indexes $\left(\mathrm{I}^{2}\right) . \mathrm{I}^{2}>50 \%$ were considered indicators of substantial heterogeneity. In the case of no significant heterogeneity, a fixed-effects model was used. A P-value $<0.05$ was considered statistically significant.

- Risk of bias across studies

Clinical heterogeneity was assessed by comparing variability among the number of samples and outcomes for survival studies; methodological heterogeneity was assessed by the risk of bias and variability in the study design. Statistical heterogeneity was also considered (HR and 95\% CI).

- Confidence in cumulative evidence

The Grading of Recommendation, Assessment, Development, and Evaluation (GRADE) instrument was used to assess evidence quality and grading of recommendation strength in the five studies included in the quantitative synthesis (11). This assessment was based on the study design, risk of bias, inconsistency, indirectness, imprecision, and other considerations. Evidence quality was characterized as high, moderate, low, or very low. The GRADE was assessed using the website http://gradepro.org.

\section{Results}

\section{- Study Selection}

In the first stage of this review, 349 studies were found in the five databases. After duplicate articles were removed, 176 remained. A screening of the titles and abstracts was carried out, and 15 records moved on to the second selection phase. In the second stage, the full-text review was then conducted on the 15 first stage selected studies, which led to the exclusion of 2 studies. After this, 13 studies fulfilled the inclusion criteria and were included in the systematic review. However, only 5 of these studies performed multivariate analysis, and the adjusted $\mathrm{HR}$ and $95 \% \mathrm{CI}$ was accessible, enabling quantitative analysis. Cohen's Kappa analysis overall score was 0.81 . Fig. 1 details this process of study selection.

- Studies Characteristics

Among the included studies, four were from China $(1,12-14)$, three were from South Korea (15-17), two from Spain $(18,19)$, and one each from Austria (20), Brazil (5), Czech Republic (21), and Japan (22). All included studies were observational studies. The year of publication of included articles ranged from 1999 to 2016. The total number of samples was 861 (775 SGNs and 86 controls). Adenoid Cystic Carcinoma (ACC) was the most studied neoplasm, followed by Mucoepidermoid Carcinoma (MEC). The follow-up period ranged from 1 to 600 months. According to the statistical analysis, the Kaplan-Meier method was used to calculate survival curves in all included studies. Univariate and multivariate Cox proportional hazard regression analysis was applied in six studies $(1,12,15-17,19)$. However, six studies did not perform Cox regression due to the absence of significance in the log-rank test and univariate analysis $(5,13,14,18,20,21)$, and in one study, the test applied was not mentioned (22). The main features and findings of the studies are presented in Table 1.

- Risk of bias within studies

Twelve studies were classified as an overall low risk of bias, and one was graded as overall moderate risk. Among the thirteen studies included, eleven studies obtained a low-risk rate on all domains (1,12-21). Two articles were graded as moderate risk on the study participation domain $(5,22)$. Of these two studies, one was graded as a high risk for statistical analysis and reporting (22). Among these two articles, there was a lack of adequate description of inclusion and exclusion criteria $(5,22)$, and the test used for survival analysis was not mentioned (22). Details about this process are shown in Table 2. The overall risk of bias assessment of the thirteen included studies is summarized in Fig. 2.

- Results of individual studies

- Demographic features

In two studies, males predominated, while nine studies demonstrated a female predilection. The F:M ratio among the included studies was 1.19:1, of which females were more affected [399] than males [335]. Regarding the age, it was observed a range from 13 to 91 years old. The most affected site was major salivary glands (456 cases) followed by the minor salivary gland (319 cases). The most affected subsite was the parotid gland (285 cases). Glands from other sites such as the paranasal sinuses, nasopharynx, nasal cavity, larynx, and the auditory canal were included in some studies. 


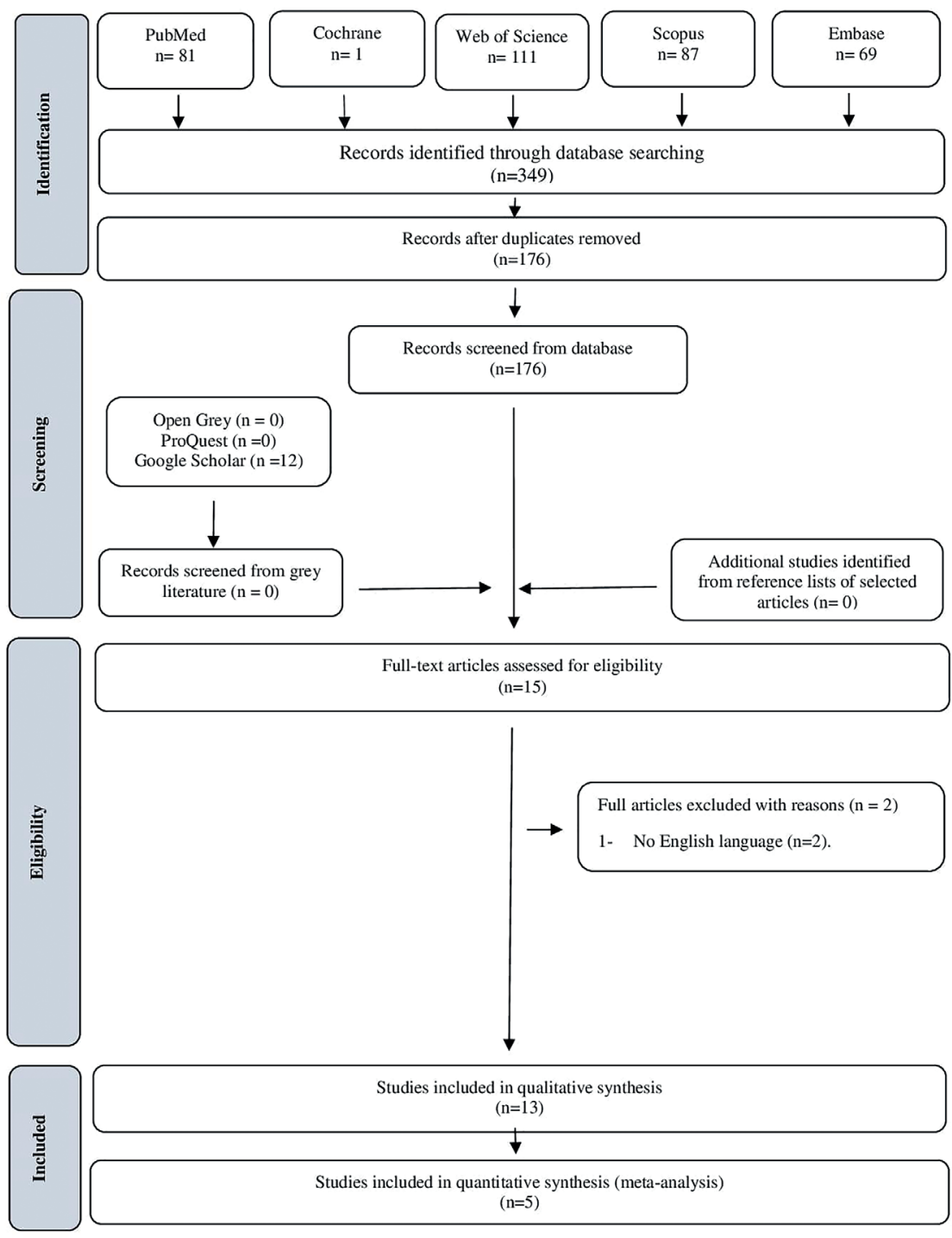

Fig. 1: Flow diagram of literature search and selection criteria.

\begin{tabular}{|c|c|c|c|c|c|c|c|}
\hline \multirow[b]{2}{*}{ Author (year) } & \multicolumn{7}{|c|}{ DOMAINS } \\
\hline & $\begin{array}{c}\text { 1. Study } \\
\text { Participation }\end{array}$ & $\begin{array}{l}\text { 2. Study } \\
\text { Attrition }\end{array}$ & $\begin{array}{l}\text { 3. Prognostic } \\
\text { Factor } \\
\text { Measurement }\end{array}$ & $\begin{array}{l}\text { 4. Outcome } \\
\text { Measurement }\end{array}$ & $\begin{array}{c}\text { 5. Study } \\
\text { Confounding }\end{array}$ & $\begin{array}{l}\text { 6. Statistical } \\
\text { Analysis and } \\
\text { Reporting }\end{array}$ & Overall Rating \\
\hline Doi et al (1999) & O & O & O & O & 0 & O & (C) \\
\hline Fonseca et al (2015) & O & $\mathrm{O}$ & 0 & 0 & 0 & O & O \\
\hline Hao et al (2009) & 0 & 0 & 0 & O & 0 & O & 0 \\
\hline Haymerle et al (2016) & 0 & 0 & 0 & 0 & 0 & 0 & 0 \\
\hline Lee et al (2012) & O & 0 & 0 & 0 & 0 & 0 & 0 \\
\hline Lequerica-Fernández et al (2007) & O & 0 & 0 & 0 & 0 & 0 & 0 \\
\hline Lequerica-Fernández et al (2011) & 0 & 0 & 0 & 0 & 0 & 0 & 0 \\
\hline Lim et al (2002) & 0 & 0 & 0 & 0 & 0 & O & 0 \\
\hline Ou Yang et al (2011) & 0 & 0 & O & 0 & 0 & 0 & 0 \\
\hline Park et al (2016) & 0 & O & 0 & 0 & 0 & 0 & 0 \\
\hline Shi et al (2007) & O & O & 0 & 0 & 0 & 0 & O \\
\hline Stárek etl al (2015) & 0 & 0 & 0 & 0 & 0 & 0 & 0 \\
\hline Zhang et al, (2005) & 0 & 0 & 0 & 0 & 0 & $\mathrm{O}$ & 0 \\
\hline
\end{tabular}

Fig. 2: Risk of bias of included studies according to the Quality In Prognosis Study (QUIPS) tool. 
Table 1: Key features and main findings of studies concerning the prognostic value immunohistochemistry detection of VEGF in SGNs.

\begin{tabular}{|c|c|c|c|c|c|}
\hline $\begin{array}{l}\text { Authors } \\
\text { (year), } \\
\text { country }\end{array}$ & $\begin{array}{l}\text { Number of } \\
\text { Samples }\end{array}$ & $\begin{array}{c}\text { Salivary gland } \\
\text { neoplasm studied }\end{array}$ & $\begin{array}{l}\text { Follow up } \\
\text { period }\end{array}$ & $\begin{array}{l}\text { Statisti- } \\
\text { cal me- } \\
\text { thod }\end{array}$ & Main results** \\
\hline $\begin{array}{c}\text { Doi et al } \\
\text { (1999), Japan }\end{array}$ & Total: 31 samples & $\begin{array}{l}\mathrm{ACC}, \mathrm{MEC}, \mathrm{ACCC}, \\
\text { SCC }\end{array}$ & $\begin{array}{c}0-60 \\
\text { months* }\end{array}$ & $\begin{array}{l}\text { Not men- } \\
\text { tioned }\end{array}$ & $\begin{array}{l}\text { The high expression of VEGF was as- } \\
\text { sociated with metastasis to lymph nodes } \\
\text { and distant sites and shortened survival. }\end{array}$ \\
\hline $\begin{array}{l}\text { Fonseca et } \\
\text { al (2015), } \\
\text { Brazil }\end{array}$ & $\begin{array}{l}\text { Total: } 132 \text { sam- } \\
\text { ples }\end{array}$ & MEC, ACNOS, ACC & $\begin{array}{l}0-600 \\
\text { months* }\end{array}$ & $\underset{\text { test }}{\text { Log-rank }}$ & $\begin{array}{l}\text { Patients with high expression of VEGF } \\
\text { has unfavorable course the disease, } \\
\text { however, there is not any association } \\
\text { between clinicopathological features } \\
\text { and VEGF expression. }\end{array}$ \\
\hline $\begin{array}{l}\text { Hao et al } \\
\text { (2009), } \\
\text { China }\end{array}$ & $\begin{array}{l}\text { Controls: } 6 \\
\text { samples } \\
\text { SGN: } 63 \text { samples } \\
\text { Total: } 69 \text { samples }\end{array}$ & $\mathrm{ACC}$ & $\begin{array}{l}6-220 \\
\text { months }\end{array}$ & $\begin{array}{l}\text { Multiva- } \\
\text { riate Cox } \\
\text { regression }\end{array}$ & $\begin{array}{l}\text { The VEGF was expressed increasingly } \\
\text { in the tissues of cases with invasion } \\
\text { and metastasis. The VEGF expression } \\
\text { was independent prognosis factors for } \\
\text { survival. }\end{array}$ \\
\hline $\begin{array}{c}\text { Haymerle } \\
\text { et al }(2016) \\
\text { Austria } \\
\end{array}$ & Total: 35 samples & $\begin{array}{c}\text { ACC, MEC,Ca ex PA, } \\
\text { ACNOS, CAC, CCC, } \\
\text { BCA } \\
\end{array}$ & $\begin{array}{l}1-412 \\
\text { months }\end{array}$ & $\begin{array}{l}\text { Log-rank } \\
\text { test }\end{array}$ & $\begin{array}{c}\text { The high expression of VEGF is related } \\
\text { with with decreased survival after re- } \\
\text { currence. }\end{array}$ \\
\hline $\begin{array}{c}\text { Lee } \text { et al } \\
\text { (2012), South } \\
\text { Korea }\end{array}$ & Total: 48 samples & $\mathrm{ACC}$ & $\begin{array}{l}4-159.1 \\
\text { months }\end{array}$ & $\begin{array}{l}\text { Multiva- } \\
\text { riate Cox } \\
\text { regression }\end{array}$ & $\begin{array}{l}\text { There is not any association between } \\
\text { VEGF and prognosis of SGNs. }\end{array}$ \\
\hline $\begin{array}{c}\text { Lequerica- } \\
\text {-Fernández } \\
\text { et al (2007), } \\
\text { Spain }\end{array}$ & Total: 66 samples & $\begin{array}{l}\text { ACC, ACNOS, TMM, } \\
\text { SCaC, ACCC, MEC }\end{array}$ & $\begin{array}{l}3-260 \\
\text { months }\end{array}$ & $\begin{array}{l}\text { Log-rank } \\
\text { test }\end{array}$ & $\begin{array}{l}\text { The VEGF expression was associ- } \\
\text { ated with neck node metastasis, worse } \\
\text { survival and poor local control of the } \\
\text { disease. }\end{array}$ \\
\hline $\begin{array}{l}\text { Lequerica- } \\
\text {-Fernández } \\
\text { et al (2011), } \\
\text { Spain }\end{array}$ & Total: 42 samples & $\begin{array}{l}\text { ACC, MEC, SDC, AC- } \\
\text { NOS, Ca ex PA, SCCa, } \\
\text { SCC, UC, PLGA, } \\
\text { ACCC, MC, BCA. }\end{array}$ & $\begin{array}{l}6-260 \\
\text { months }\end{array}$ & $\begin{array}{l}\text { Multiva- } \\
\text { riate Cox } \\
\text { regression }\end{array}$ & $\begin{array}{l}\text { The VEGF expression is an independent } \\
\text { prognostic factor in parotid gland can- } \\
\text { cer. High expression of VEGF is related } \\
\text { with shortened survival. }\end{array}$ \\
\hline $\begin{array}{l}\text { Lim et al } \\
\text { (2002), South } \\
\text { Korea }\end{array}$ & Total: 45 samples & $\begin{array}{l}\text { ACC, SDC, MEC, } \\
\text { PLGA, Ca ex PA, AC- } \\
\text { NOS, }\end{array}$ & $\begin{array}{l}5-150 \\
\text { months }\end{array}$ & $\begin{array}{l}\text { Multiva- } \\
\text { riate Cox } \\
\text { regression }\end{array}$ & $\begin{array}{l}\text { The VEGF expression was significantly } \\
\text { correlated with age, tumor size, lymph } \\
\text { node metastasis, clinical stage, perineu- } \\
\text { ral invasion, vascular invasion, recur- } \\
\text { rence and survival. VEGF may be an } \\
\text { independent prognostic factor for SGNs. }\end{array}$ \\
\hline $\begin{array}{l}\text { Ou Yang et } \\
\text { al (2011), } \\
\text { China }\end{array}$ & $\begin{array}{c}\text { Controls: } 40 \\
\text { samples } \\
\text { SGN: } 70 \text { samples } \\
\text { Total: } 110 \\
\text { samples } \\
\end{array}$ & MEC & $\begin{array}{l}1-60 \\
\text { months }\end{array}$ & $\begin{array}{l}\text { Log-rank } \\
\text { test }\end{array}$ & $\begin{array}{l}\text { The expression of VEGF was } \\
\text { significantly associated with tumor dif- } \\
\text { ferentiation, size metastasis, relapse and } \\
\text { poor survival, but was not correlated } \\
\text { lymph node metastasis and metastasis. }\end{array}$ \\
\hline $\begin{array}{c}\text { Park et al } \\
\text { (2016), South } \\
\text { Korea }\end{array}$ & Total: 68 samples & $\mathrm{ACC}$ & $\begin{array}{l}4-263 \\
\text { months }\end{array}$ & \begin{tabular}{|l|} 
Multiva- \\
riate Cox \\
regression \\
\end{tabular} & $\begin{array}{l}\text { High expression of VEGF was indepen- } \\
\text { dent prognostic factor related with poor } \\
\text { overall survival. }\end{array}$ \\
\hline $\begin{array}{l}\text { Shi et al } \\
\text { (2007), } \\
\text { China }\end{array}$ & $\begin{array}{c}\text { Controls: } 20 \\
\text { samples } \\
\text { SGN: } 75 \text { samples } \\
\text { Total: } 95 \text { samples }\end{array}$ & MEC & $\begin{array}{l}3-252 \\
\text { months }\end{array}$ & $\begin{array}{l}\text { Univa- } \\
\text { riete Cox } \\
\text { regression }\end{array}$ & $\begin{array}{l}\text { There is not any association between } \\
\text { VEGF and prognosis of SGNs. }\end{array}$ \\
\hline $\begin{array}{l}\text { Stárek et } \\
\text { al (2015), } \\
\text { Czech } \\
\text { Republic }\end{array}$ & Total: 20 samples & $\mathrm{ACC}$ & $\begin{array}{l}5-282 \\
\text { months }\end{array}$ & $\underset{\text { test }}{\text { Log-rank }}$ & $\begin{array}{l}\text { There is not any association between } \\
\text { VEGF expression and prognosis of } \\
\text { SGNs. }\end{array}$ \\
\hline $\begin{array}{l}\text { Zhang et } \\
\text { al (2005), } \\
\text { China }\end{array}$ & $\begin{array}{l}\text { Controls: } 20 \\
\text { samples } \\
\text { SGN: } 80 \text { samples } \\
\text { Total: } 100 \\
\text { samples }\end{array}$ & $\mathrm{ACC}$ & $\begin{array}{l}24-144 \\
\text { months }\end{array}$ & $\begin{array}{l}\text { Multiva- } \\
\text { riate Cox } \\
\text { regression }\end{array}$ & $\begin{array}{l}\text { The immunoreactivity of VEGF was } \\
\text { correlated with tumor size, clinical } \\
\text { stage, vascular invasion, recurrence, } \\
\text { and distant metastasis. The VEGF ex- } \\
\text { pression had an independent prognostic } \\
\text { effect on overall survival. }\end{array}$ \\
\hline
\end{tabular}

Abbreviations: Acinic Cell Carcinoma (ACCC); Pleomorphic Adenoma (PA ); Whartin Tumor (WT); Mucoepidermoid Carcinoma (MEC); Carcinoma Ex pleomorphic Adenoma (Ca ex PA ); Adenoid Cystic Carcinoma (ACC); Poorly Differentiated Carcinoma (PDC); Epithelial Myoepithelial Carcinoma (EMC); Adenocarcinoma NOS (ACNOS); Salivary Duct Carcinoma (SDC); Small Cell Carcinoma (SCC); Carcinosarcoma (CS); Adenosquamous Carcinoma; Oncocitoma (OnC); Myoepithelial Carcinoma (MC); Neuroendocrine Carcinoma (NC); Cystadeno Carcinoma (CAC); Oncocytic Carcinoma (OC); Undifferentiated Carcinoma (UC); Renal Cell Carcinoma (RCC); Squamous Cell Carcinoma (SCca); Basal Adenocarcinoma (BA).

*Data extracted from the Kaplan-Meier survival curves; **All results showed $P</=0.05$. 
Table 2: Risk of bias categorized as (H) High risk, (L) Low Risk and (M) Moderate Risk according to recommendations from Cochrane Methods Prognosis group utilizing the Quality in Prognosis Studies (QUIPS) tool.

\begin{tabular}{|c|c|c|c|c|c|c|c|c|c|c|c|c|c|}
\hline & 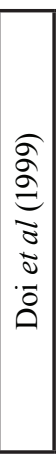 & $\begin{array}{l}\approx \\
0 \\
0 \\
0 \\
z \\
\tilde{0} \\
\tilde{d} \\
0 \\
0 \\
0 \\
0\end{array}$ & 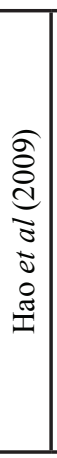 & 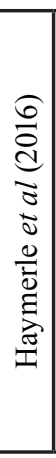 & 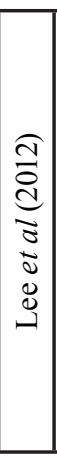 & 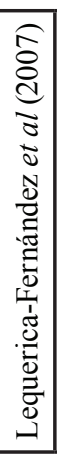 & 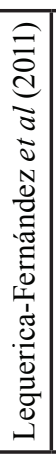 & 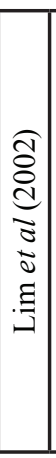 & 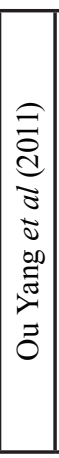 & 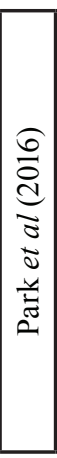 & 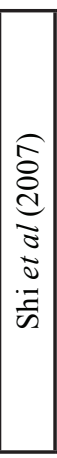 & 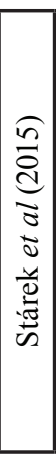 & 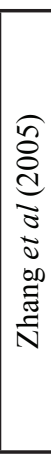 \\
\hline \multicolumn{14}{|c|}{ Study Participation } \\
\hline Adequate participation in the study by eligible persons & $\mathrm{M}$ & $\mathrm{M}$ & $\mathrm{L}$ & $\mathrm{L}$ & $\mathrm{L}$ & $\mathrm{L}$ & $\mathrm{L}$ & $\mathrm{L}$ & $\mathrm{L}$ & $\mathrm{L}$ & $\mathrm{L}$ & $\mathrm{L}$ & $\mathrm{L}$ \\
\hline Description of the source population or population of interest & $\mathrm{M}$ & $\mathrm{L}$ & $\mathrm{L}$ & $\mathrm{L}$ & $\mathrm{L}$ & $\mathrm{L}$ & $\mathrm{L}$ & $\mathrm{L}$ & $\mathrm{L}$ & $\mathrm{L}$ & $\mathrm{L}$ & $\mathrm{L}$ & $\mathrm{L}$ \\
\hline Description of the baseline study sample & $\mathrm{L}$ & $\mathrm{L}$ & $\mathrm{L}$ & $\mathrm{L}$ & $\mathrm{L}$ & $\mathrm{L}$ & $\mathrm{L}$ & $\mathrm{L}$ & $\mathrm{L}$ & $\mathrm{L}$ & $\mathrm{L}$ & $\mathrm{L}$ & $\mathrm{L}$ \\
\hline Adequate description of the sampling frame and recruitment & $\mathrm{L}$ & $\mathrm{L}$ & $\mathrm{L}$ & $\mathrm{L}$ & $\mathrm{L}$ & $\mathrm{L}$ & $\mathrm{L}$ & $\mathrm{L}$ & $\mathrm{L}$ & $\mathrm{L}$ & $\mathrm{L}$ & $\mathrm{L}$ & $\mathrm{L}$ \\
\hline Adequate description of the period and place of recruitment & $\mathrm{L}$ & $\mathrm{M}$ & $\mathrm{L}$ & $\mathrm{L}$ & $\mathrm{L}$ & $\mathrm{L}$ & $\mathrm{L}$ & $\mathrm{L}$ & $\mathrm{L}$ & $\mathrm{L}$ & $\mathrm{L}$ & $\mathrm{L}$ & $\mathrm{L}$ \\
\hline Adequate description of inclusion and exclusion criteria & $\mathrm{H}$ & $\mathrm{H}$ & $\mathrm{L}$ & $\mathrm{H}$ & $\mathrm{L}$ & $\mathrm{L}$ & $\mathrm{L}$ & $\mathrm{M}$ & $\mathrm{L}$ & $\mathrm{M}$ & $\mathrm{L}$ & $\mathrm{M}$ & $\mathrm{M}$ \\
\hline \multicolumn{14}{|l|}{ Study Attrition } \\
\hline Adequate response rate for study participants & $\mathrm{L}$ & $\mathrm{L}$ & $\mathrm{L}$ & $\mathrm{L}$ & $\mathrm{L}$ & $\mathrm{L}$ & $\mathrm{L}$ & $\mathrm{L}$ & $\mathrm{L}$ & $\mathrm{L}$ & $\mathrm{L}$ & $\mathrm{L}$ & $\mathrm{L}$ \\
\hline $\begin{array}{l}\text { Description of attempts to collect information on } \\
\text { dropped out }\end{array}$ & $\mathrm{L}$ & $\mathrm{L}$ & $\mathrm{L}$ & $\mathrm{L}$ & $\mathrm{L}$ & $\mathrm{L}$ & $\mathrm{L}$ & $\mathrm{L}$ & & $\mathrm{L}$ & $\mathrm{L}$ & $\mathrm{L}$ & $\mathrm{L}$ \\
\hline Reasons for loss to follow-up are provided & $\mathrm{L}$ & $\mathrm{L}$ & & $\mathrm{L}$ & $\mathrm{L}$ & $\mathrm{L}$ & $\mathrm{L}$ & \begin{tabular}{|l|l}
$\mathrm{L}$ \\
\end{tabular} & $\mathrm{L}$ & $\mathrm{L}$ & $\mathrm{L}$ & $\mathrm{L}$ & $\mathrm{L}$ \\
\hline Adequate description of participants lost to follow-up & $\mathrm{L}$ & $\mathrm{L}$ & $\mathrm{L}$ & $\mathrm{L}$ & $\mathrm{L}$ & $\mathrm{L}$ & $\mathrm{L}$ & $\mathrm{L}$ & $\mathrm{L}$ & $\mathrm{L}$ & $\mathrm{L}$ & $\mathrm{L}$ & $\mathrm{L}$ \\
\hline $\begin{array}{l}\text { between participants who com- } \\
\text { not }\end{array}$ & $\mathrm{L}$ & $\mathrm{L}$ & $\mathrm{L}$ & $\mathrm{L}$ & $\mathrm{L}$ & $\mathrm{L}$ & $\mathrm{L}$ & $\mathrm{L}$ & $\mathrm{L}$ & $\mathrm{L}$ & $\mathrm{L}$ & $\mathrm{L}$ & $\mathrm{L}$ \\
\hline \multicolumn{14}{|c|}{ Prognostic Factor Measurement } \\
\hline A clear definition or description of the PF is provided & $\mathrm{L}$ & $\mathrm{L}$ & $\mathrm{L}$ & $\mathrm{L}$ & $\mathrm{L}$ & $\mathrm{L}$ & $\mathrm{L}$ & $\mathrm{L}$ & $\mathrm{L}$ & $\mathrm{L}$ & $\mathrm{L}$ & $\mathrm{L}$ & $\mathrm{L}$ \\
\hline Method of PF measurement is adequately valid and reliable & $\mathrm{L}$ & $\mathrm{L}$ & $\mathrm{L}$ & $\mathrm{L}$ & $\mathrm{L}$ & $\mathrm{L}$ & $\mathrm{L}$ & $\mathrm{L}$ & $\mathrm{L}$ & $\mathrm{L}$ & $\mathrm{L}$ & $\mathrm{L}$ & $\mathrm{L}$ \\
\hline Continuous variables are reported or appropriate cut points are used & $\mathrm{L}$ & $\mathrm{L}$ & $\mathrm{L}$ & $\mathrm{L}$ & $\mathrm{L}$ & $\mathrm{L}$ & $\mathrm{L}$ & $\mathrm{L}$ & $\mathrm{L}$ & $\mathrm{L}$ & $\mathrm{L}$ & $\mathrm{L}$ & $\mathrm{L}$ \\
\hline $\begin{array}{l}\text { The method and setting of measurement of PF is the same for all } \\
\text { study participants }\end{array}$ & $\mathrm{L}$ & $\mathrm{L}$ & $\mathrm{L}$ & $\mathrm{L}$ & $\mathrm{L}$ & $\mathrm{L}$ & $\mathrm{L}$ & $\mathrm{L}$ & $\mathrm{L}$ & $\mathrm{L}$ & $\mathrm{L}$ & $\mathrm{L}$ & $\mathrm{L}$ \\
\hline Adequate proportion of the study sample has complete data for the PF & $\mathrm{L}$ & $\mathrm{L}$ & $\mathrm{L}$ & $\mathrm{L}$ & $\mathrm{L}$ & $\mathrm{L}$ & $\mathrm{L}$ & $\mathrm{L}$ & $\mathrm{L}$ & $\mathrm{L}$ & $\mathrm{L}$ & $\mathrm{L}$ & $\mathrm{L}$ \\
\hline Appropriate methods of imputation are used for missing PF data & $\mathrm{L}$ & $\mathrm{L}$ & $\mathrm{L}$ & $\mathrm{L}$ & $\mathrm{L}$ & $\mathrm{L}$ & $\mathrm{L}$ & $\mathrm{L}$ & $\mathrm{L}$ & $\mathrm{L}$ & $\mathrm{L}$ & $\mathrm{L}$ & $\mathrm{L}$ \\
\hline \multicolumn{14}{|c|}{ Outcome Measurement } \\
\hline A clear definiti & $\mathrm{L}$ & $\mathrm{L}$ & $\mathrm{L}$ & $\mathrm{L}$ & $\mathrm{L}$ & $\mathrm{L}$ & $\mathrm{L}$ & $\mathrm{L}$ & $\mathrm{L}$ & $\mathrm{L}$ & $\mathrm{L}$ & $\mathrm{L}$ & $\mathrm{L}$ \\
\hline Method of outcome measurement used is adequately valid & $\mathrm{L}$ & $\mathrm{L}$ & $\mathrm{L}$ & $\mathrm{L}$ & $\mathrm{L}$ & $\mathrm{L}$ & $\mathrm{L}$ & $\mathrm{L}$ & $\mathrm{L}$ & $\mathrm{L}$ & $\mathrm{L}$ & $\mathrm{L}$ & $\mathrm{L}$ \\
\hline $\begin{array}{l}\text { The method and setting of outcome measurement is the same for all } \\
\text { study participants }\end{array}$ & $\mathrm{L}$ & $\mathrm{L}$ & $\mathrm{L}$ & $\mathrm{L}$ & $\mathrm{L}$ & $\mathrm{L}$ & $\mathrm{L}$ & $\mathrm{L}$ & $\mathrm{L}$ & $\mathrm{L}$ & $\mathrm{L}$ & $\mathrm{L}$ & $\mathrm{L}$ \\
\hline \multicolumn{14}{|c|}{ Study Confounding } \\
\hline All impo & $\mathrm{M}$ & $\mathrm{M}$ & $\mathrm{M}$ & $\mathrm{M}$ & $\mathrm{M}$ & $\mathrm{M}$ & $\mathrm{M}$ & $\mathrm{M}$ & $\mathrm{M}$ & $\mathrm{M}$ & $\mathrm{M}$ & $\mathrm{M}$ & $\mathrm{M}$ \\
\hline Clear definitions of the important confounders measured are provided & $\mathrm{L}$ & $\mathrm{L}$ & $\mathrm{L}$ & $\mathrm{L}$ & $\mathrm{L}$ & $\mathrm{L}$ & $\mathrm{L}$ & $\mathrm{L}$ & $\mathrm{L}$ & $\mathrm{L}$ & $\mathrm{L}$ & $\mathrm{L}$ & $\mathrm{L}$ \\
\hline Measurement of all important confounders is adequately valid and reliable & $\mathrm{L}$ & $\mathrm{L}$ & $\mathrm{L}$ & $\mathrm{L}$ & $\mathrm{L}$ & $\mathrm{L}$ & $\mathrm{L}$ & $\mathrm{L}$ & $\mathrm{L}$ & $\mathrm{L}$ & $\mathrm{L}$ & $\mathrm{L}$ & $\mathrm{L}$ \\
\hline $\begin{array}{l}\text { The method and setting of confounding measurement are the same } \\
\text { for all study participants }\end{array}$ & $\mathrm{L}$ & $\mathrm{L}$ & $\mathrm{L}$ & $\mathrm{L}$ & $\mathrm{L}$ & $\mathrm{L}$ & $\mathrm{L}$ & $\mathrm{L}$ & $\mathrm{L}$ & $\mathrm{L}$ & $\mathrm{L}$ & $\mathrm{L}$ & $\mathrm{L}$ \\
\hline $\begin{array}{l}\text { Appropriate methods are used if imputation is used for missing con- } \\
\text { founder data }\end{array}$ & $\mathrm{L}$ & $\mathrm{L}$ & $\mathrm{L}$ & $\mathrm{L}$ & $\mathrm{L}$ & $\mathrm{L}$ & $\mathrm{L}$ & $\mathrm{L}$ & $\mathrm{L}$ & $\mathrm{L}$ & $\mathrm{L}$ & $\mathrm{L}$ & $\mathrm{L}$ \\
\hline Important potential confounders are accounted for in the study design & $\mathrm{L}$ & $\mathrm{L}$ & $\mathrm{L}$ & $\mathrm{L}$ & $\mathrm{L}$ & $\mathrm{L}$ & $\mathrm{L}$ & $\mathrm{L}$ & $\mathrm{L}$ & $\mathrm{L}$ & $\mathrm{L}$ & $\mathrm{L}$ & $\mathrm{L}$ \\
\hline Important potential confounders are accounted for in the analysis & $\mathrm{L}$ & $\mathrm{L}$ & $\mathrm{L}$ & $\mathrm{L}$ & $\mathrm{L}$ & $\mathrm{L}$ & $\mathrm{L}$ & $\mathrm{L}$ & $\mathrm{L}$ & $\mathrm{L}$ & $\mathrm{L}$ & $\mathrm{L}$ & $\mathrm{L}$ \\
\hline \multicolumn{14}{|c|}{ Statistical Analysis and Reporting } \\
\hline $\begin{array}{l}\text { Sufficient presentation of data to assess the adequacy of the analytic } \\
\text { strategy }\end{array}$ & $\mathrm{H}$ & $\mathrm{L}$ & $\mathrm{L}$ & $\mathrm{L}$ & $\mathrm{L}$ & $\mathrm{L}$ & $\mathrm{L}$ & $\mathrm{L}$ & $\mathrm{L}$ & $\mathrm{L}$ & $\mathrm{L}$ & $\mathrm{L}$ & $\mathrm{L}$ \\
\hline $\begin{array}{l}\text { Strategy for model building is appropriate and is based on a concep- } \\
\text { tual framework or model }\end{array}$ & $\mathrm{H}$ & $\mathrm{L}$ & $\mathrm{L}$ & $\mathrm{L}$ & $\mathrm{L}$ & $\mathrm{L}$ & $\mathrm{L}$ & $\mathrm{L}$ & $\mathrm{L}$ & $\mathrm{L}$ & $\mathrm{L}$ & $\mathrm{L}$ & $\mathrm{L}$ \\
\hline The selected statistical model is adequate for the design of the study & $\mathrm{H}$ & $\mathrm{L}$ & $\mathrm{L}$ & $\mathrm{L}$ & $\mathrm{L}$ & $\mathrm{L}$ & $\mathrm{L}$ & $\mathrm{L}$ & $\mathrm{L}$ & $\mathrm{L}$ & $\mathrm{L}$ & $\mathrm{L}$ & $\mathrm{L}$ \\
\hline There is no selective reporting of results & $\mathrm{H}$ & $\mathrm{L}$ & $\mathrm{L}$ & $\mathrm{L}$ & $\mathrm{L}$ & $\mathrm{L}$ & $\mathrm{L}$ & $\mathrm{L}$ & $\mathrm{L}$ & $\mathrm{L}$ & $\mathrm{L}$ & $\mathrm{L}$ & $\mathrm{L}$ \\
\hline
\end{tabular}


- Microscopic features

All included studies provided a histologic classification of their samples, and they graded the tumors according to the classification of the World Health Organization (WHO) (23). Adenoid cystic carcinoma was by far the most studied lesion followed by mucoepidermoid carcinoma. The histologic patterns of ACC included were cribriform, tubular, solid, and intermediate. MEC were classified as low-grade, intermediate-grade, and high-grade. Eight studies reported the presence of perineural invasion in a sample of 153 patients. Vascular invasion was reported in four studies affecting 50 patients.

- Clinical features

The presence of clinical symptoms, such as numbness, pain, bleeding, and paresthesia, was observed in 96 patients. Regarding the clinical stages, 300 patients were classified on clinical stage I/II and 245 patients in clinical stage III/V. -

- Overall analysis of VEGF expression

VEGF was expressed both in the cytoplasm and in cell membranes with different intensities. Immunoreactivity was present in 87\% [676] of cases analyzed. Despite heterogeneity among the histopathologic types of SGNs analyzed, some studies demonstrated that the VEGF was associated with shortened overall survival in patients with SGNs. On the other hand, some studies showed a lack of association between VEGF expression and any prognostic factor in patients with SGNs.

The tumor that showed the greatest association with increased VEGF expression was ACC. In the studies that analyzed ACC isolated, it was shown that overexpression of VEGF was significantly associated with decreased survival and was correlated with tumor size, clinical stage, vascular invasion, recurrence, and distant metastasis $(P<0.05)(4,12,17)$. VEGF was not associated with age, tumor sites, and patients 'gender. Despite this, two studies failed to find an association between VEGF overexpression and survival $(14,15)$. Some studies correlated the VEGF expression and MEC, and it was observed that the expression levels of VEGF were significantly related to tumor differentiation, size, and relapse $(P<0.05)$, but were not correlated with lymph node metastasis, distant metastasis, or overall survival $(13,14)$.

Among the studies that pooled different types of SGNs, there was a strong association of clinical and pathologic factors and VEGF expression, such as clinical stage, tumor size, age, vascular invasion, perineural invasion, survival, and recurrence $(P<0.05)(16,18-20)$. The isolated prognostic factor strongly associated with different levels of VEGF expression was metastasis. In general, there was a significant association between immunohistochemistry overexpression of VEGF and shortened survival.

- Synthesis of results

Five studies assessed the OS of SGN samples using Kaplan-Meier curves, which generated Hazard Ratios (HR). The meta-analysis used the adjusted hazard ratio $+95 \%$ CI was derived from the multivariate analysis from included articles. The pooled HR was $5.37(95 \%$ CI: $2.67-10.83 ; P=0.00001 ; \mathrm{I}^{2}=0 \%$ ). Low heterogeneity was observed between the studies, with an $\mathrm{I}^{2}$ of $0 \%(P=0.82)$, leading to the decision of the fixed-effect model. As shown in Fig. 3, higher immunohistochemical expression of VEGF in SGNs may favor shortened overall survival. One study found a significant association between VEGF's high immunohistochemical expression and poor overall survival, and it was an independent prognostic factor (17).

- Risk of bias across studies

Regarding the risk of bias across studies, the selected studies used similar methods, which reduced the possibility of misinterpretation. According to studies design, they were considered homogeneous, which was confirmed by the low heterogeneity that led to the fixed effect choice.

- Confidence in cumulative evidence

According to GRADE analysis, the quality of the evidence for survival analysis was moderate. Due to this, it is reasonable to suggest moderate confidence in estimating the outcomes. The CI difference in each study was the main factor responsible for the limited quality of evidence on the imprecision assessment (Table 3).

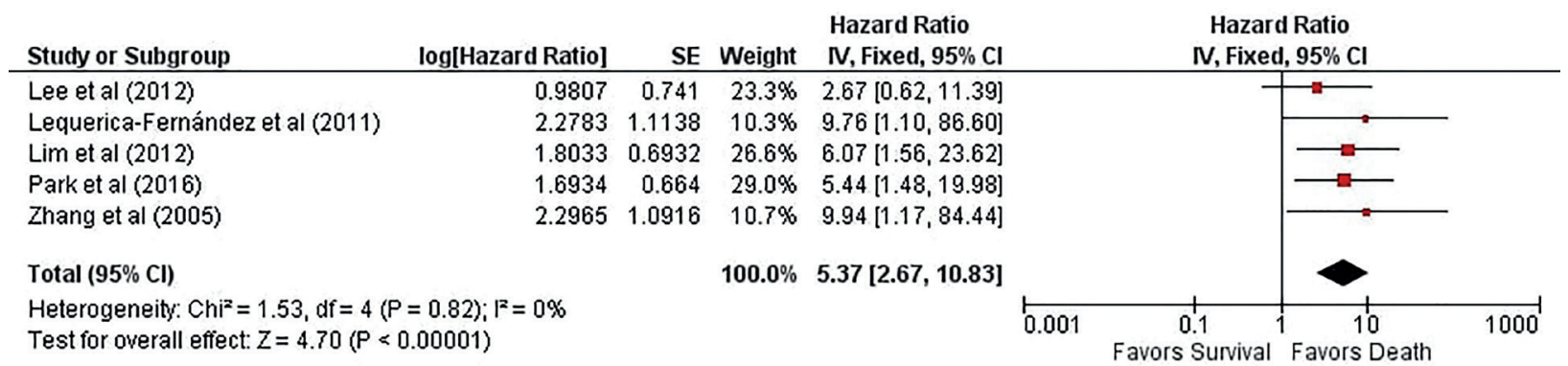

Fig. 3: High expression of VEGF is significantly associated with shorten survival. Forest plot of hazard ratio for survival comparing patients with overexpression of VEGF in the malignant salivary gland tumors compared with those with low immunoreactivity. The meta-analysis revealed that VEGF was associated with poorly survival (HR: 5.37, 95\% CI: 2.67-10.83, P $<.00001$ ). The diamond represents the pooled HR performed by the fixed-effect model. 
Table 3: Table representing prognostic studies included in the meta-analysis assessing Grading of Recommendation, Assessment, Development, and Evaluation (GRADE) instrument. Question: Is there any association between immunohistochemical detection of VEGF and overall survival in patients with malignant salivary gland neoplasms?

\begin{tabular}{|c|c|c|c|c|c|c|c|c|}
\hline \multicolumn{7}{|c|}{ Quality assessment } & \multirow[t]{2}{*}{ Quality } & \multirow{2}{*}{$\begin{array}{l}\text { Impor- } \\
\text { tance }\end{array}$} \\
\hline $\begin{array}{c}\text { \# of } \\
\text { studies }\end{array}$ & $\begin{array}{l}\text { Study } \\
\text { design }\end{array}$ & $\begin{array}{c}\text { Risk of } \\
\text { bias }\end{array}$ & $\begin{array}{l}\text { Inconsis- } \\
\text { tency }\end{array}$ & Indirectness & $\begin{array}{c}\text { Impreci- } \\
\text { sion }\end{array}$ & $\begin{array}{c}\text { Other consider- } \\
\text { ations }\end{array}$ & & \\
\hline 5 & $\begin{array}{l}\text { Observa- } \\
\text { tional stud- } \\
\text { ies }\end{array}$ & $\begin{array}{l}\text { Not seri- } \\
\text { ous }\end{array}$ & Not serious & Not serious & Not serious & $\begin{array}{l}\text { Strong association. } \\
\text { all plausible re- } \\
\text { sidual confound- } \\
\text { ing would suggest } \\
\text { spurious effect, } \\
\text { while no effect } \\
\text { was observed; } \\
\text { dose response } \\
\text { gradient }\end{array}$ & $\begin{array}{c}\bigoplus \bigoplus \bigoplus 囚 \\
\text { MODERATE }\end{array}$ & $\begin{array}{c}\text { We are } \\
\text { moderately } \\
\text { confident in } \\
\text { the effect } \\
\text { estimate }\end{array}$ \\
\hline
\end{tabular}

High quality: We are very confident that the true effect lies close to that of the estimate of the effect. Moderate quality: We are moderately confident in the effect estimate: The true effect is likely to be close to the estimate of the effect, but there is a possibility that it is substantially different. Low quality: Our confidence in the effect estimate is limited: The true effect may be substantially different from the estimate of the effect. Very low quality: We have very little confidence in the effect estimate: The true effect is likely to be substantially different from the estimate of effect.

\section{Discussion}

The role of VEGF in tumor progression and metastasis has been studied in several malignant neoplasms, including head and neck cancer $(1,4,5)$. Despite this, the data of the prognostic value of VEGF in patients with SGNs remains unclear. Some studies have reported that VEGF may be useful as an independent prognostic factor for these tumors $(1,15-17,19)$. On the other hand, some authors suggested that there is not any significant association between VEGF and SNGs $(5,14,15,21)$. Due to these controversial findings, we performed a systematic review and meta-analysis from the literature to assess the association of VEGF by immunohistochemical analysis and the overall survival of patients with SGNs. Data were extracted from thirteen studies with 775 samples of SGNs tissue analyzed by immunohistochemistry. The combined results showed that the positivity for VEGF in the tissue of these patients was associated with shortened survival. Moreover, the presence of VEGF was associated with clinical stage, perineural invasion, vascular invasion, tumor size, poor local control of the disease, and recurrence $(1,13,16,18)$. In addition, publication bias examination and heterogeneity assumption tested by the $\mathrm{I}^{2}$ metric support our findings, although, due to CI difference in each study, the quality of the evidence for survival analysis was moderate.

The prognostic factor more associated with VEGF overexpression was metastasis $(1,12,13,16,18,22)$. Metastasis is an event uncommon on salivary gland tumors, and its mechanism is still unclear in these lesions. However, it is well established that patients with local and distant metastasis have a poor prognosis (4). It is not fully understood how VEGF can promote metastasis in SNGs, but this relation was largely studied in other tumors (24). The activation of VEGF family members and their receptors aid in the escape of the immune system, migration, and extracellular matrix invasion promoting metastasis (24). In this systematic review, it was observed that patients with high levels of VEGF expression presented distant or lymph node metastasis and poor prognosis.

Interestingly, four studies did not find any association between VEGF and prognosis in SGNs $(5,14,15,21)$. Fonseca and colleagues pooled a total of 132 formalin-fixed, paraffin-embedded tissue of SGNs (5). They failed to identify an association between clinicopathologic features and VEGF expression, probably due to the small sample size of malignant SGNs analyzed in the study. This finding was confirmed by other authors, although the majority of samples analyzed were composed of tumors of clinical stages I and II and lower histologic grade (14). SGNs are a heterogeneous group of lesions with different molecular mechanisms involved in its progression. The angiogenesis may be affected by each tumor's inherent features, leading to different expression of VEGF.

The prognostic value of VEGF has already been evaluated in other types of cancer, such as colorectal and non-small cell lung cancer (NSCLC) $(25,26)$. In a previous study, 1.428 colorectal cancer patients from 13 clinical trials were pooled in a meta-analysis, and it was observed that immunohistochemical detection of VEGF predicted poor survival in these patients (25). Also, VEGF's immunohistochemical expression was associated with poor prognosis for NSCLC patients, including patients with clinical stage I (26). However, VEGF was not significantly correlated with survival for patients with lung adenocarcinoma (26). Interestingly, our results showed a close association of VEGF overexpression in adenocarcinoma of salivary glands and poor survival $(16,18-20)$. To the best of our knowledge, this current systematic review and meta-analysis was the first to find an association between VEGF and prognosis in SGNs. These findings confirm the results 
of previous studies and lead to the necessity of more investigation of the role of VEGF in SGNs.

It is necessary to highlight some limitations in this review. First, although only one technique was used in all studies (immunohistochemistry), the variation in antibodies utilized in each study may lead to differences in the quality and intensity of staining results and interpretation of results. Second, the studies analyzed a limited sample size of SGNs, and some studies were excluded from the meta-analysis due to lack of appropriate information such as multivariate analysis, adjusted HR, or $95 \%$ CI. Further, the high difference in the $95 \%$ CI of included studies of the meta-analysis leading to high standard error and different weights in each study, which indicate that the meta-analysis should be interpreted cautiously. Therefore, we suggest more well-designed primary studies to increase the quality of evidence.

\section{Conclusions}

In conclusion, this systematic review and meta-analysis demonstrated that VEGF overexpression in patients with malignant salivary gland neoplasms has prognostic value and is associated with poor overall survival and may be useful in clinical practice.

\section{References}

1. Zhang J, Peng B, Chen X. Expressions of nuclear factor kappaB, inducible nitric oxide synthase, and vascular endothelial growth factor in adenoid cystic carcinoma of salivary glands: correlations with the angiogenesis and clinical outcome. Clin Cancer Res. 2005;11:7334-43.

2. Maulik N, Das DK. Redox signaling in vascular angiogenesis. Free Radic Biol Med. 2002;33:1047-60.

3. Kieser A, Weich H, Brandner G. Mutant p53 potentiates protein kinase $\mathrm{C}$ induction of vascular endothelial growth factor expression. Oncogene. 1994;9:963-9.

4. Zang J, Li C, Zhao LN, Shi M, Zhou YC, Wang JH, et al. Prognostic value of vascular endothelial growth factor in patients with head and neck cancer: A meta-analysis. Head Neck. 2013;35:1507-14. 5. Fonseca FP, Basso MP, Mariano FV, Kowalski LP, Lopes MA, Martins MD, et al. Vascular endothelial growth factor immunoexpression is increased in malignant salivary gland tumors. Ann Diagn Pathol. 2015;19:169-74.

6. Moher D, Liberati A, Tetzlaff J, Altman DG; PRISMA Group. Preferred reporting items for systematic reviews and meta-analyses: the PRISMA statement. PLoS Med. 2009;6:e1000097.

7. Ouzzani M, Hammady H, Fedorowicz Z, Elmagarmid A. Rayyan - a web and mobile app for systematic reviews. Syst Rev. 2016;5:210

8. Hayden JA van der Windt DA, Cartwright JL, Côté P, Bombardier C. Assessing bias in studies of prognostic factors. Ann Intern Med. 2013;158:280-6.

9. Hayden JA, Côté P, Bombardier C. Evaluation of the quality of prognosis studies in systematic reviews. Ann Intern Med. 2006;144:427-37.

10. Leeflang MM, Deeks JJ, Takwoingi Y, Macaskill P. Cochrane diagnostic test accuracy reviews. Syst Rev. 2013;2:82.

11. Balshem H, Helfand M, Schünemann HJ, Oxman AD, Kunz R, Brozek J, et al. GRADE guidelines: 3 . Rating the quality of evidence. J Clin Epidemiol. 2011;64:401-6.

12. Hao L, Xiao-lin N, Qi C, Yi-ping Y, Jia-quan L, Yan-ning L.
Nerve growth factor and vascular endothelial growth factor: retrospective analysis of 63 patients with salivary adenoid cystic carcinoma. Int J Oral Sci. 2010;2:35-44.

13. Ou Yang KX1, Liang J, Huang ZQ. Association of clinicopathologic parameters with the expression of inducible nitric oxide synthase and vascular endothelial growth factor in mucoepidermoid carcinoma. Oral Dis. 2011;17:590-6.

14. Shi L, Chen XM, Wang L, Zhang L, Chen Z. Expression of caveolin-1 in mucoepidermoid carcinoma of the salivary glands: correlation with vascular endothelial growth factor, microvessel density, and clinical outcome. Cancer. 2007;109:1523-31.

15. Lee SK, Kwon MS, Lee YS, Choi SH, Kim SY, Cho KJ, et al. Prognostic value of expression of molecular markers in adenoid cystic cancer of the salivary glands compared with lymph node metastasis: a retrospective study. World J Surg Oncol. 2012;11:266.

16. Lim JJ, Kang S, Lee MR, Pai HK, Yoon HJ, Lee JI, et al. Expression of vascular endothelial growth factor in salivary gland carcinomas and its relation to p53, Ki-67 and prognosis. J Oral Pathol Med. 2003;32:552-61.

17. Park S, Nam SJ, Keam B, Kim TM, Jeon YK, Lee SH, et al. VEGF and Ki-67 Overexpression in Predicting Poor Overall Survival in Adenoid Cystic Carcinoma. Cancer Res Treat. 2016;48:518-26. 18. Lequerica-Fernández P, Astudillo A, de Vicente JC. Expression of vascular endothelial growth factor in salivary gland carcinomas correlates with lymph node metastasis. Anticancer Res. 2007;27:3661-6. 19. Lequerica-Fernández P, Peña I, Villalaín L, Rosado P, de Vicente JC. Carcinoma of the parotid gland: developing prognostic indices. Int J Oral Maxillofac Surg. 2011;40:821-8.

20. Haymerle G, Schneider S, Harris L, Häupl T, Schopper C, Pammer J, et al. Minor salivary gland carcinoma: a review of 35 cases. Eur Arch Otorhinolaryngol. 2016;273:2717-26.

21. Stárek I, Salzman R, Kučerová L, Skálová A, Hauer L. Expression of VEGF-C/-D and lymphangiogenesis in salivary adenoid cystic carcinoma. Pathol Res Pract. 2015;211:759-65.

22. Doi R, Kuratate I, Okamoto E, Ryoke K, Ito H. Expression of p53 oncoprotein increases intratumoral microvessel formation in human salivary gland carcinomas. J Oral Pathol Med. 1999;28:259-63.

23. El-Naggar AK, Chan JKC, Takata T, Grandis JR, Slootweg PJ. The fourth edition of the head and neck World Health Organization blue book: editors' perspectives. Hum Pathol. 2017;66:10-12.

24. Ceci C, Atzori MG, Lacal PM, Graziani G. Role of VEGFs/ VEGFR-1 Signaling and its Inhibition in Modulating Tumor Invasion: Experimental Evidence in Different Metastatic Cancer Models. Int J Mol Sci. 2020;21:1388.

25. Zong S, Li H, Shi Q, Liu S, Li W, Hou F. Prognostic significance of VEGF-C immunohistochemical expression in colorectal cancer: A meta-analysis. Clin Chim Acta. 2016;458:106-14.

26. Jiang H, Shao W, Zhao W. VEGF-C in non-small cell lung cancer: meta-analysis. Clin Chim Acta. 2014;427:94-9.

\section{Acknowledgements}

This study was financed in part by the São Paulo Research Foundation (FAPESP 2018/19922-9). The authors are also grateful to the Brazilian National Council for Scientific and Technological Development $(\mathrm{CNPq})$ for student scholarship. Adriana Franco Paes Leme is researcher fellow funded by the Brazilian National Council for Scientific and Technological Development (CNPq).

\section{Funding}

None declared.

\section{Conflict of interest}

The authors declare that there is no conflict of interest that could bias the results of this manuscript.

\section{Ethics}

Given that this is a systematic review, no ethical approval was required. 
Authors contributions

ESS and AFPL were responsible by conception and design of the study. ESS and AGCN performed data collection and analysis of results. AFPL analyzed the obtained results when mutual agreement between other authors was not reached. JCR performed the GRADE analysis and provided additional interpretation of data when necessary. ESS drafted the main manuscript text, and AGCN, JCR and AFPL revised the manuscript. The final version of the manuscript was read and approved by all authors. All authors are available to ensure the questions related to any issue in this article. 